\title{
Elastic and Inelastic Collisions of Swarms
}

\author{
Dieter Armbruster \\ School of Mathematical and Statistical Sciences, Arizona State University \\ Stephan Martin \\ Department of Mathematics, Imperial College London \\ Andrea Thatcher \\ School of Mathematical and Statistical Sciences, Arizona State University, Tempe, AZ \\ $U S A$
}

\begin{abstract}
Scattering interactions of swarms in potentials that are generated by an attraction-repulsion model are studied. In free space, swarms in this model form a well-defined steady state describing the translation of a stable formation of the particles whose shape depends on the interaction potential. Thus, the collision between a swarm and a boundary or between two swarms can be treated as (quasi)-particle scattering. Such scattering experiments result in internal excitations of the swarm or in bound states, respectively. In addition, varying a parameter linked to the relative importance of damping and potential forces drives transitions between elastic and inelastic scattering of the particles. By tracking the swarm's center of mass, a refraction rule is derived via simulations relating the incoming and outgoing directions of a swarm hitting the wall. Iterating the map derived from the refraction law allows us to predict and understand the dynamics and bifurcations of swarms in square boxes and in channels.
\end{abstract}

Keywords: Swarm models, swarm scattering, swarm reflection, attraction-repulsion model

PACS: 05.65.+b, 45.50.Jf, 87.23.Cc

Email addresses: armbruster@asu.edu (Dieter Armbruster), smartin.research@gmail.com (Stephan Martin), andee.thatcher@gmail.com (Andrea Thatcher)

Preprint submitted to Elsevier

November 22, 2016

(C) 2016. This manuscript version is made available under the Elsevier user license http://www.elsevier.com/open-access/userlicense/1.0/ 


\section{Introduction}

The collective behavior of many self-propelled particles has been the focus of research: applications range from fish, bird, and locust swarms $[1,9,11,19]$ to computer bots [23], pedestrian and crowd behavior [18], markets [3], and opinion dynamics [17].

Parallel to these applications, there are simulation models based on agents [25], analytic models based on ordinary differential equations [12], physical models relating to phase transitions [13] and large scale continuum models based on partial differential equations $[16,7]$.

Mostly, the analysis of these swarm models focusses on the onset of swarming, the different swarming states and the stability and bifurcations under parameter variations. This paper assumes fully formed swarms (flocks) and studies their collisional behavior and scattering properties. As a model swarming system, we use the attraction-repulsion model introduced by [14] which treats a swarm in analogy to multi-particle interactions based on a potential in atomic or nuclear physics.

Extending the physics analogy, we study the scattering interaction between flocks. Since a flock is a well-defined translational steady state and all particles are governed by an interaction potential, the collision between a flock and a boundary or between two flocks can be looked at as (quasi)particle scattering. We will show that such scattering results in internal excitations of the flocks in the former case or in bound states for the latter. We continue this approach to multiple scattering characterizing the motion of a flock in boxes and channels.

This study is a companion study to the analysis of the interaction of the Vicsek swarm model [25] with the boundaries of finite domains. Additional references more specific to the analysis of the Vicsek model can be found there [2].

\subsection{The attraction-repulsion model}

The model [14] describes particles that move according to Newton's law in a potential that is generated by the mutual interactions between all particles depending on their relative distances:

$$
\begin{aligned}
\frac{d x_{i}}{d t} & =v_{i} \\
\frac{d v_{i}}{d t} & =\left(\alpha-\beta\left|v_{i}\right|^{2}\right) v_{i}-\lambda \nabla_{x_{i}} \sum_{j \neq i} U\left(x_{i}-x_{j}\right), \quad i=1 . . N
\end{aligned}
$$


where $N$ is the total number of particles and $x_{i}, v_{i}$ are the position and velocity of the $i^{\text {th }}$ particle. Here, $\alpha v_{i} \geq 0$ is the self-propulsion force and $\beta\left|v_{i}\right|^{2} \geq 0$ is a Raleigh type friction force. The potential strength $\lambda$ introduced by Carillo et al. [4] reflects the impact of the potential forces relative to the self-propelling and damping forces. It will also be useful to think about $\lambda$ as the timescale associated to the potential forces relative to the timescales of the self-propelling velocity $v=\sqrt{\frac{\alpha}{\beta}} . \lambda$ large implies oscillations that are faster than the swarm speed whereas small $\lambda$ implies slow modulations.

The pairwise interaction force in Equation (1) is generally defined to be attracting for large distances and repelling for short distances to represent biological systems [22]. One typical example is the scaled Morse potential

$$
U(x)=k(|x|), \quad k(r)=C \exp (-r / l)-\exp (-r),
$$

where $C$ and $l$ with $C l^{2} \geq 1$ are the ratios of the attractive and repulsive strength and the typical lengths of attraction and repulsion, respectively. Other potentials have been considered such as the quasi-Morse potential $[6,8]$ or Log-Newtonian potential [15].

Ignoring the Morse potential, the self-acceleration force $\alpha v_{i}$ and the selfdeceleration force $\beta\left|v_{i}\right|^{2} v_{i}$ tend to balance out and the system attains a constant (nontrivial) speed $\left|v_{F}\right|=\sqrt{\alpha / \beta}$ which has no influence on the orientation of the velocities. The orientation of the velocities depends on the initial conditions.

Since we are interested in the interplay between swarms and walls, we fix all the parameters in the system with the exception of $\lambda$. Specifically, we choose $l=\frac{3}{4}$ and $C=\frac{10}{9}$, placing us into the parameter region labelled catastrophic in the analysis presented in [14]. For this case, the pairwise interaction potential has a well defined minimum (see Figure 1a). The catastrophic region is characterized by the fact that increasing the number of particles causes the spacing between particles to reduce, keeping the volume constant. In this parameter region, the stationary solutions of the system depend on the values of $\alpha$ and $\beta$. For larger values of $\alpha / \beta$, particles disperse while at relatively lower values, coherent structures form. When $\alpha$ is small, a core-free mill state emerges (Figure 1c)) [10]. Since particles travel at a non-zero uniform speed, the interaction potential is not strong enough to support them too close to the center and an empty core forms. The size of the empty core depends on $N$. As $N \rightarrow \infty$, the core collapses since we are 


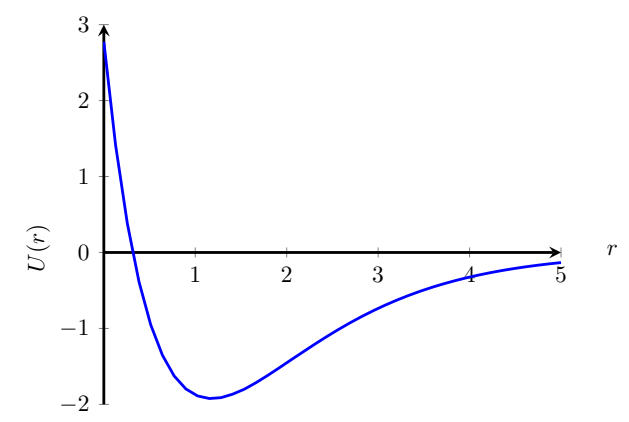

(a) Morse potential for $C=10 / 9$ and $l=3 / 4$.

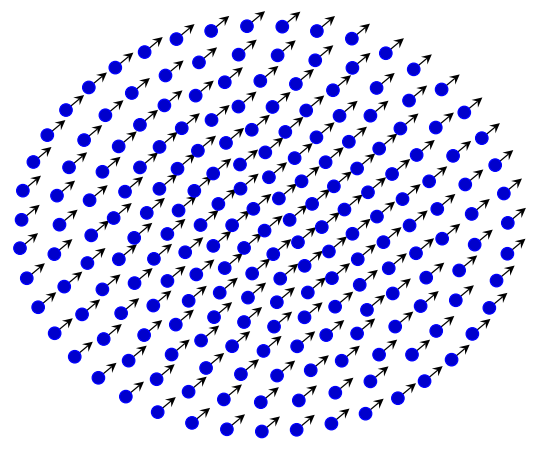

(b) Flock

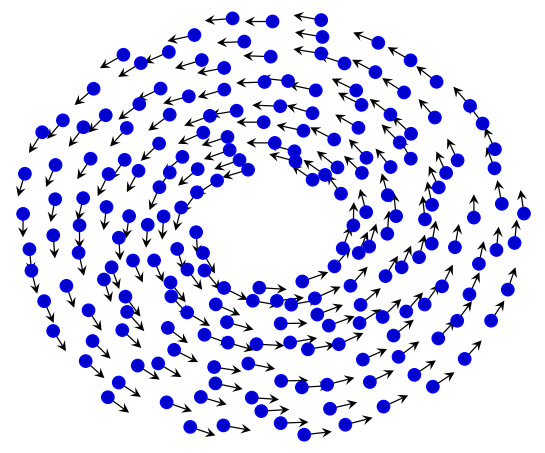

(c) Single Mill

Figure 1: a) Typical Morse potential in the catastrophic region, b) typical flock solution, c) typical mill solution. 
in the catastrophic region [14].

As $\alpha$ increases, the milling formation cannot be sustained and a typical steady state solution is the flock (Figure 1b)) [21] where all particles travel with a unified velocity $\sqrt{\alpha / \beta}$ and maintain a minimal relative distance.

When the kinetic energy greatly surpasses the strength of the interaction potential, the swarm breaks apart and no aggregation can be found.

We focus our attention on the scattering interaction of the flock solution with boundaries and with other flocks where a flock is analytically defined as:

Definition 1. A flock solution of the particle model (1) is a spatial configuration $\hat{x}$ with zero net interaction force on every particle, that translates at a uniform velocity $v_{F} \in \mathbb{R}^{2}$ with $\left|v_{F}\right|=\sqrt{\alpha / \beta}$, hence $\left(x_{i}(t), v_{i}(t)\right)=$ $\left(\hat{x}_{i}+t v_{F}, v_{F}\right)$. The spatial configuration $\hat{x}$ is a stationary state to the firstorder interacting particle system

$$
\frac{d x_{i}}{d t}=-\sum_{j \neq i} \nabla_{i} U\left(x_{i}-x_{j}\right) .
$$

Notice that the flock is not rotationally invariant. A small rotation about the center of mass of the flock still satisfies Eq. (3) but has a different spatial representation. Carillo et al. [5] provide a stability analysis for this flock solution and show that the flock is capable of withstanding small perturbations.

\subsection{Simulation Setup}

We simulate the behavior of flocks satisfying Definition 1 and analyze the results of the simulations via concepts from dynamical systems.

We use a 4th order Runge-Kutta method to compute the numerical approximation to system (1). For all considered values of the potential strength

$\lambda$, the eigenvalues of the system lie within the stability region for a time step of $\Delta t=0.1$. We note that very long simulation runs produce reproducible results, indicating that even with the addition of reflecting walls, the algorithm is still stable.

When reaching the reflecting boundary, a single particle will experience specular reflection and its velocity is updated as $v_{\text {new }}=v_{\text {old }}-2\left(v_{\text {old }} \cdot \vec{n}\right) \vec{n}$ where $\vec{n}$ is the normal vector to the boundary. 
Other numerical methods to represent the evolution of particles are possible. For instance, the authors in [14] implement a multistep method AdamsBashforth-Moulton. However, when implementing this method, a particle interacting with the wall between time steps $n-1$ and $n$ is updated by the numerical method which uses the time steps $n-3$ to $n$ to calculate the reflected velocity at time step $n+1$. This leads to a discontinuity when the velocity is updated at $n+1$ and the resulting path of the particle does not reflect specularly. We chose the 4th order Runge Kutta 4th since it is a onestep method that creates no discontinuity and allows the particle to reflect specularly.

For all simulations, we initialize the system with a flock where $v_{F}$ is dependent on the initial heading $\theta_{0} \in[0,2 \pi)$ where the angle $\theta_{0}$ is calculated from the positive $x$-axis

$$
v_{F}\left(\theta_{0}\right)=\sqrt{\alpha / \beta}\left[\cos \left(\theta_{0}\right), \sin \left(\theta_{0}\right)\right] .
$$

The parameter choices for each simulation (unless stated otherwise) are: ratio between strength of repulsion and attraction $C=10 / 9$, ratio between the length of repulsion and attraction $l=3 / 4$, number of particles $N=100$, friction force $\beta=5$, acceleration force $\alpha=1$, potential pre-factor $\lambda=25$, initial heading $\theta_{0}=0$, and $\Delta t=0.1$. We average all findings over 10 spatial rotations and run most simulations for 500 time units to 10000 time units. We run the same experiments with $N=200$ and find similar results.

Simulations were run using MATLAB on $96 \mathrm{gb}$ RAM and $2 \operatorname{Intel}(\mathrm{R})$ $\mathrm{Xeon}(\mathrm{R}) \mathrm{CPU}$ E5-2620 0 @ 2.00GHz processors and the average time for 5000 time units took 1200 seconds.

The paper is structured as follows: Section 2.1 considers the collision of a flock with a wall, focussing on the internal dynamics generated by the interaction (section 2.1.1) and the refraction law for the path of the center of mass of the flock in Section 2.1.2. Using the refraction law as an iterated map, we analyze the dynamics of flocks in a channel (section 2.2.1) and a square box (section 2.2.2). Applying principle component analysis to a single flock on wall interaction, we find that the internal dynamics of a swarm can be well understood as a damped elastic oscillation characterized by a single eigenfunction (section 3). Swarm on swarm collisions are discussed in section 4 where we show that the two colliding swarms either pass each other, leading to a change in the relative angles of their direction of motion or to the swarms joining to become one or if they have an almost head-on collision, they will form a bound state and become a mill solution. 


\section{Scattering a Single Swarm at Boundaries}

We seek to understand multiple swarm collisions within a bounded domain as a sequence of single wall collisions. We start with a single collision of one swarm with a boundary.

\subsection{Single Wall Collision}

\subsubsection{Individual Particle Motion}

We consider the attraction-repulsion model (1) in an unbounded halfspace in $R^{2}$ with a specularly reflecting wall at $x=L$. We initialize a flock to collide with the wall with initial headings $\theta_{0} \in[0, \pi / 2)$. When $\theta_{0}=\pi / 2$, the flock translates upward, i.e. is parallel to the wall and hence never interacts with the wall. We note that in free space, a flock will remain intact and continue on its set trajectory for all time.

Our focus here are the individual particles in a swarm during a head on collision event, i.e. $\theta_{0}=0$. We find two regimes: one in which all particles hit the wall, the swarm reflects specularly and its internal order is unchanged and another one where the swarm only partially hits the wall, the swarm exhibits a damped oscillatory excitation and the internal structure of the swarm becomes completely mixed.

The first regime is valid for small $\lambda$. Figure 2a) and c) shows that all particles hit the wall and bounce back specularly. The second regime applies for large $\lambda$ : Approximately the leading $2 / 3$ of the particles physically hit the wall and bounce back. The other particles experience a changed interaction potential due to the change in velocity and position of the leading particles. The changed potential creates a differential slowing down within the swarm, influencing the trailing particles to turn around before they hit the wall. This is illustrated in figure $2 \mathrm{~b}$ ): the maroon colored particles on the left of the swarm will not hit the wall but will turn around earlier. The resulting reflected swarm experiences strong internal mixing. Figure 2d) shows that the initially leading particles and the initially trailing particles mix strongly.

To characterize the internal remixing of the swarm after its collision, we determine the initial position in the swarm along the $x$-axis that separates particles directly hitting the wall and to those that do not (see Fig. 2b).

Figure 3a) shows that for $\lambda<13$ the position that separates the two behaviors is at the trailing edge of the swarm, i.e. all particles hit the wall, whereas for $\lambda>13$ that point moves approximately linearly forward. 


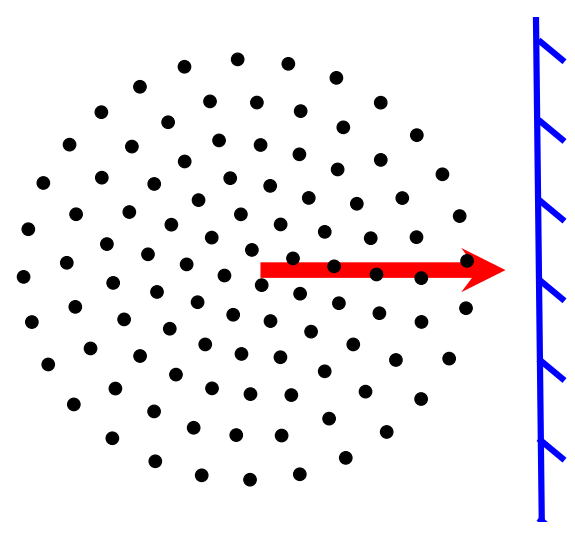

(a) Initial State: $\lambda=1$

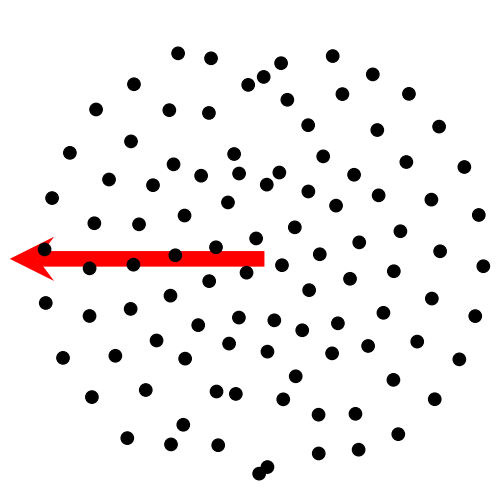

(c) End State: $\lambda=1$

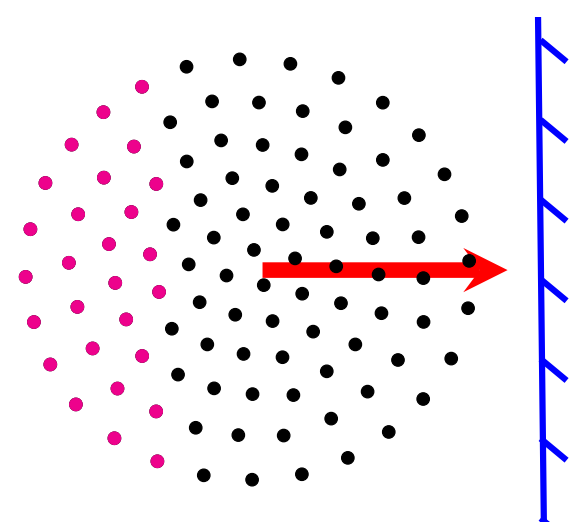

(b) Initial State: $\lambda=25$

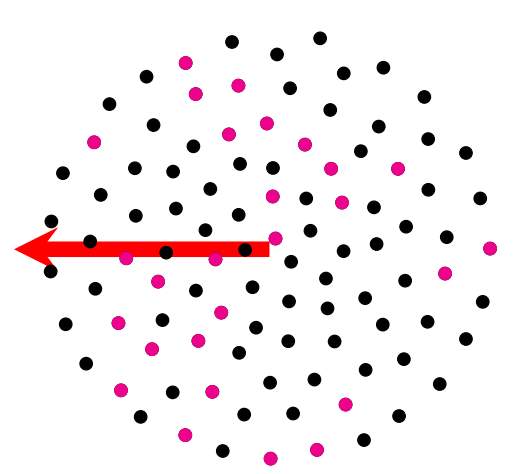

(d) End State: $\lambda=25$

Figure 2: Initial and end states for a swarm impacting a vertical wall at the right. Particles that will directly interact with the wall are colored black and particles that do not interact with the wall are colored maroon. a) All particles hit the wall for $\lambda=1$, b) the trailing particles turn around before they hit the wall for $\lambda=25$. c) and d) depict the end states i.e., the location of particles of both types after the swarms reflect. Note that for $\lambda=25$ the trailing edge of the swarm will not hit the wall but the particles in that group will be spread in an apparently random way within the swarm after the collision. 


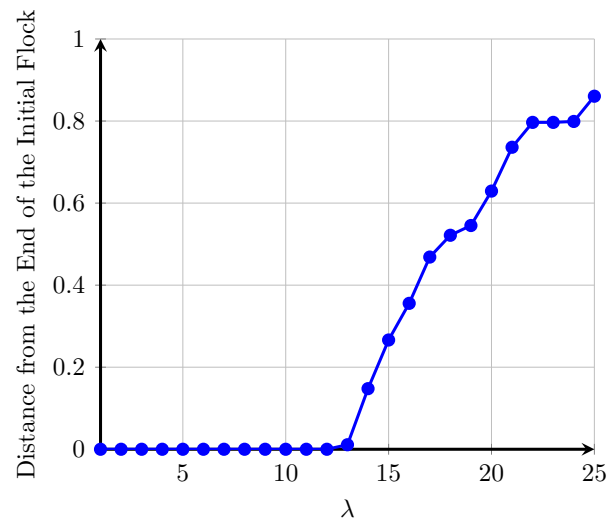

(a) Particle position

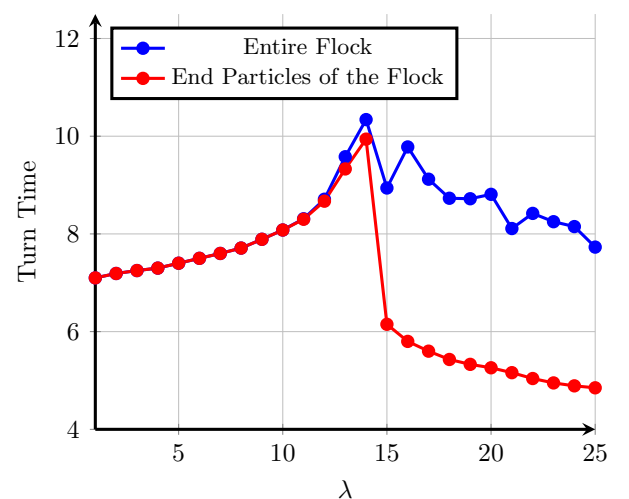

(b) Turn Time

Figure 3: a) Position of the first particle in the direction of motion of the flock that turns around without hitting the wall. The position is measured as a fraction of the total diameter of the swarm. b) Turning time for the most distant particles (red) and for the ones that turn last (black),

Figure $3 \mathrm{~b}$ ) presents the turning time (blue curve) when the last particle of the swarm turns around (i.e. $v_{x}$ changes direction) as a function of $\lambda$. The red curve shows the the time for the most distant particle from the wall to turn around. Both values coincide and increase with $\lambda$ until $\lambda \approx 13$. Increasing $\lambda$ further, the most distant particle is no longer the last to turn.

Studying the kinetic energies in the $\mathrm{x}$ and $\mathrm{y}$ direction (Figure 4) helps clarify the swarm-wall interaction. For $\lambda \approx 1$, the swarm reflection is totally specular, all the kinetic energy is in the $x$ direction and it stays constant throughout the wall interaction.

As $\lambda$ increases, the swarm slows down (decreasing total kinetic energy) and is compressed, i.e. kinetic energy is converted into potential elastic energy. As the potential energy is released, the swarm undergoes internal damped oscillations and kinetic energy in both the $x$ and $y$ directions is involved. As a result, the flock experiences a breathing or pulsing motion. We further examine this motion in section 3 where we perform a Proper Orthogonal Decomposition of the simulation data.

\subsubsection{Refraction Law}

To characterize the behavior of the swarm as a whole, we track the center of mass of the flock and measure the outgoing angle of its trajectory as a function of the incoming angle $\theta_{0}$. Figure $5 a$ a) illustrates the definition of the 


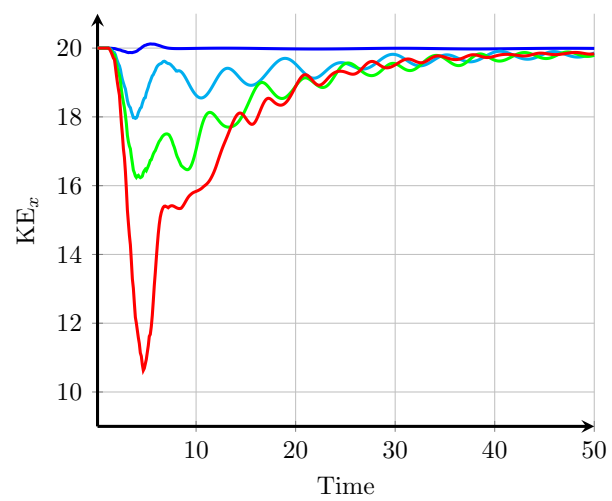

(a) Kinetic Energy: $\frac{1}{2} \sum v_{x}^{2}$

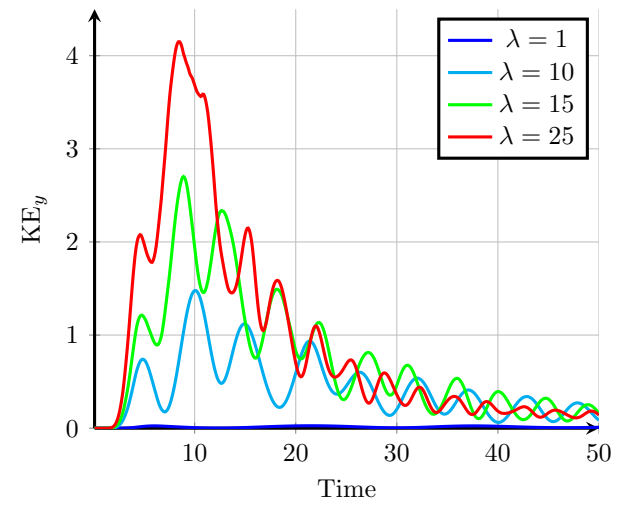

(b) Kinetic Energy: $\frac{1}{2} \sum v_{y}^{2}$

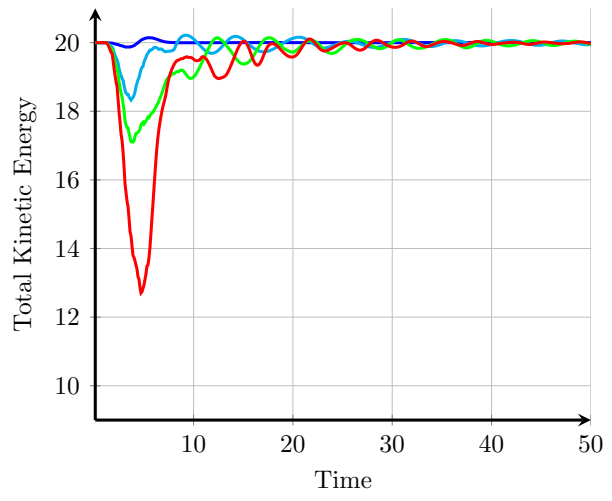

(c) Total Kinetic Energy

Figure 4: Kinetic energy transfer from the $x$-direction to the $y$-direction for several $\lambda$ values. For $\lambda=1$ the flock reflects specularly, ie. the kinetic energy stays constant and is only coming fro $v_{x}$. As $\lambda$ increases, the kinetic energy during the collision converts to potential energy creating a damped pulsating motion that involves motion in the $\mathrm{x}$ and $\mathrm{y}$ directions. 
measurement of the outgoing angle. If the trajectory of the center of mass is specularly reflected, the incoming and outgoing angles are equal. If the outgoing angle is different than the incoming, the wall interaction does not follow the reflection law but rather a refraction law.

Figure 5b) shows the outgoing angles as a function of the incoming angle and $\lambda .{ }^{1}$ We see that as $\lambda$ increases the outgoing angle aligns more with the wall. Figure 5c) emphasizes this result by plotting the difference between the outgoing angle and the initial angle $\theta_{0}$. For small $\lambda$ the flock reflects specularly while as $\lambda$ increases, this difference increases. In particular, when only a fraction of the particles hit the wall $(\lambda>13$, see section 2.1 .1$)$, the outgoing swarm strongly aligns with the wall.

Summarizing, we find two types of reflection at the wall:

1. The swarm as a whole interacts elastically with the wall, characterized by specular reflection of all particles leading to a specular reflection of the swarm.

2. The swarm as a whole interacts inelastically with the wall, caused by the fact that some particles do not hit the wall when they turn around. As a result, the swarm exhibits damped oscillatory excitations and its outgoing direction tends to align with the wall.

The behavior of the swarm bifurcates from type 1 to type 2 at about $\lambda=13$.

\subsection{Multiple Reflections}

We assume that, after a collision with a wall, the swarm fully stabilizes to the free equilibrium solution before it hits the next wall. Thus, we can consider the refraction law at a wall as a one-dimensional map, relating the incoming angle to an outgoing angle and analyzing multiple reflections as iterated maps. We study their limiting behavior as the number of wall interactions goes to infinity. Convergence of the iterated map to a fixed point or to a periodic orbit corresponds to a stable periodic or period-doubled trajectory of the swarm in the geometrical domain. An unstable fixed point corresponds to an unstable periodic orbit of the swarm. We restrict our study to both a channel and a square geometry.

\footnotetext{
${ }^{1}$ Note: For $\theta_{0}=0$, the flock has equal chance of reflecting upward or downward. When averaging over the spatial rotations, we take the absolute value of the outgoing angle for each rotation. Whether the flock aligns upward or downward is irrelevant at $\theta_{0}=0$.
} 


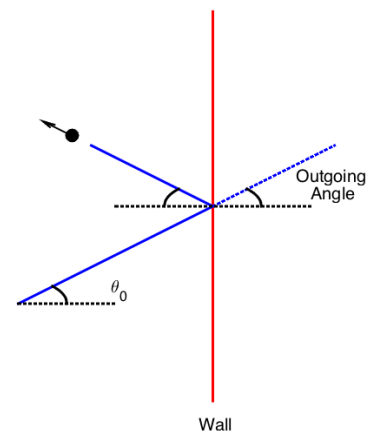

(a) Calculating Outgoing Angles

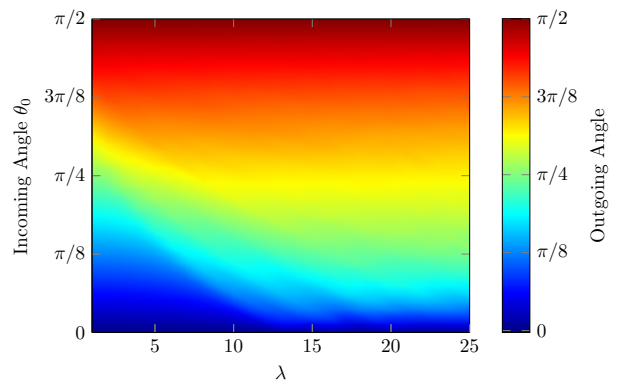

(b) Refraction Laws

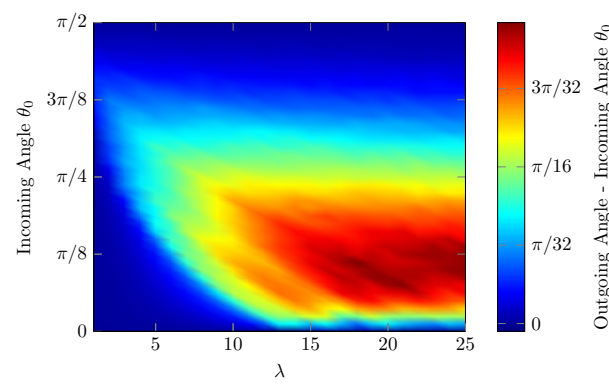

(c) Difference Between Outgoing Angles and $\theta_{0}$

Figure 5: Refraction law of a flock interacting with a wall. a) Schematic definition of the outgoing angle. b) Outgoing angle as a function of incoming angle and $\lambda$. c) The outgoing angle minus the initial angle $\theta_{0} . \lambda$ grid spacing is $0.5, \theta$ grid spacing is $\frac{\pi}{32}$. 


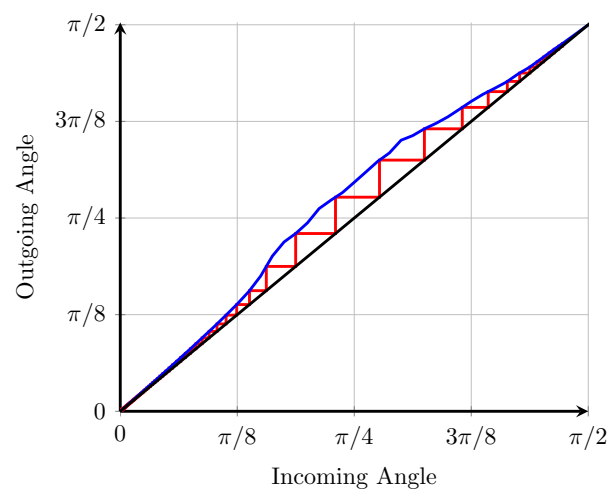

(a) $\lambda=5$

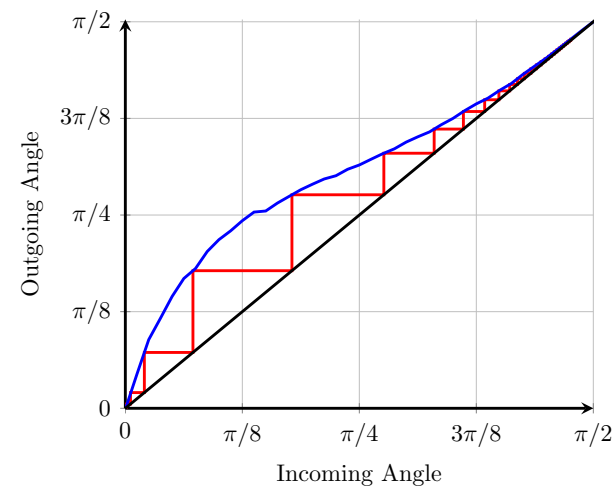

(b) $\lambda=25$

Figure 6: One-dimensional maps and the staircase diagrams for the outgoing angles as a function of the incoming angles. a) For $\lambda=5$ the equilibrium at zero is weakly unstable and the equilibrium at $\pi / 2$ is weakly stable. b) For $\lambda=25$ the equilibrium at 0 is linearly unstable (i.e. has eigenvalue $>1$ ).

\subsubsection{Channel}

In a vertical channel, the outgoing angle of a flock leaving a sidewall is the incoming angle for the next wall collision. Figure 6 shows two such maps for the outgoing angle as a function of the incoming angle with an associated staircase diagram. The staircase diagram shows a trajectory under the iteration of the map for $\lambda=5$ and $\lambda=25$. In both cases, there are two equilibria: $\theta_{e 1}=0$ and $\theta_{e 2}=\frac{\pi}{2}$, the former being unstable and the latter being stable. Notice that both equilibria for $\lambda=5$ have a slope of 1, i.e. an eigenvalue of 1 (within the accuracy of the numerical simulation). Nevertheless, the graph indicates that $\theta=0$ is unstable and $\theta=\frac{\pi}{2}$ is stable. We call these cases weakly stable (unstable).

In Figure 6 the Channel width is chosen large enough, such that the swarm will equilibrate completely before it hits the next wall. Formally this is the case for the width to be infinite, but for a width of 100 units, the swarm is very well organized when it hits. If we reduce the channel width, the swarm is not given enough time to equilibrate before the next wall collision. For really small width, the perturbations generated by the wall interactions are additive and eventually causes the flock to break apart. When the flock breaks apart and the milling state is not stable i.e. for small $\lambda$ values [10], particles move randomly. For larger $\lambda$ where the milling state is stable, they

coalesce to that state. The influence of the noise generated by fast repetition 


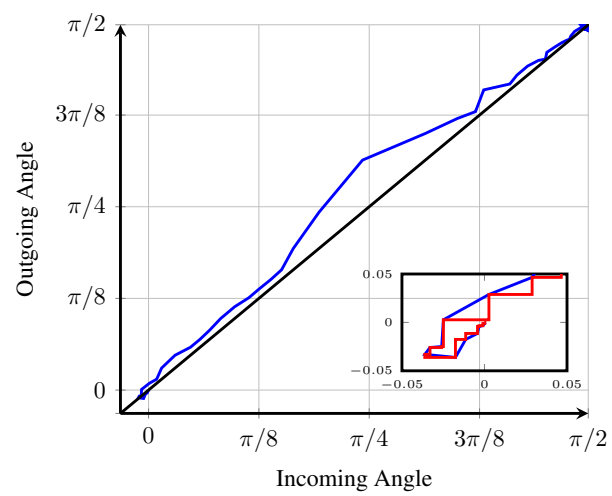

(a)

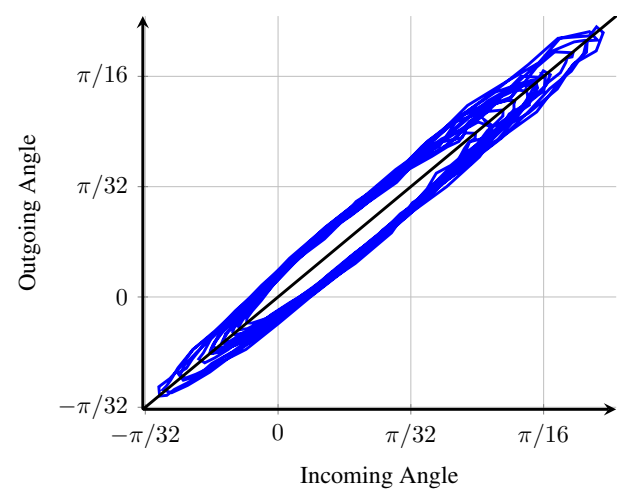

(b)

Figure 7: Swarm trajectory for a narrow channel (width 14 units) and $\lambda=5$ : a) weakly unstable equilibrium at $\theta=0$. Inset- the flock starts to drift downward but switches direction and drifts upward. b) Sample trajectory of a swarm that is stuck in a horizontal periodic orbit for 5000 time units (different initial condition than in a).

of the wall interaction is seen when $\lambda$ is small. For a starting angle close to zero, i.e. the unstable equilibrium, the instability is very weak and hence susceptible to noise. The inset in figure 7a) shows a case where the swarm bounces back and forth between the sidewalls without settling on an upward or downward movement before eventually aligning with the channel in the upwards direction. Noise may even trap the swarm in a horizontal back and forth motion that does not align with the channel for a long simulation time (Figure 7b) ).

\subsubsection{Square}

Prediction of the dynamics based on the iterated refraction map. We characterize the trajectory of a swarm in a square by the outgoing angle $\eta \in[-\pi / 2, \pi / 2]$ calculated from the boundary in the direction that the swarm is moving. For clockwise rotation, $\eta<0$ while for counterclockwise rotation $\eta>0$. Notice that by simple geometry, the outgoing angle $\eta_{n}$ after the $n^{\text {th }}$ wall interaction leads to an incoming angle at the next wall of the form $\pi / 2-\eta_{n}$. Then, for specular reflection in a square, the sequence of outgoing angles is $\eta_{0} \rightarrow \frac{\pi}{2}-\eta_{0} \rightarrow \eta_{0}$ for any starting angle $\eta_{0}$, filling the square densely with periodic orbits.

Assuming a square that is big enough and a swarm that hits a boundary is far enough away from the lateral boundaries, the internal excitations decay 
before the swarm hits the next wall and we can, again, assume that the refraction law discussed in section 2.1.2 holds and remains the same at every boundary. Given the geometry of the domain, we iterate the refraction map twice and study its fixed points and stability to predict the swarm behavior in a square box. We then simulate actual swarms in such a box and compare the prediction with the simulation results.

Figure 8 shows the twice iterated map $\eta_{n} \rightarrow \eta_{n+2}$ for different values of $\lambda$. A fixed point of this map is a periodic trajectory of the swarm in the square domain. Figure 8a) shows that for small $\lambda$ there are three fixed points: $\eta_{e 1}=0$ corresponding to a motion parallel to a boundary, $\eta_{e 2}$ slightly less than $\frac{\pi}{4}$ corresponding to a symmetric orbit connecting approximately the midpoints of the sides of the square, and $\eta_{e 3}=\frac{\pi}{2}$ corresponding to a motion parallel to a boundary and orthogonal to the one associated with $\eta_{e 1}$. Inspection of the staircase diagrams shows that the motions parallel to the boundary are attracting while the symmetric trajectory is unstable.

Near $\lambda=6$ (figure 8(b)) the reflection law becomes specular, i.e. every angle results in a periodic orbit. In terms of a bifurcation, we have a degenerate bifurcation leading from an unstable fixed point in the interior of the interval $[0, \pi / 2]$ to a stable fixed point shown Figure $8 \mathrm{c}$ ) and d) via a completely linear map. As a result of the bifurcation, the motions parallel to the boundaries become unstable. The bifurcation leading to the instability of the orbits parallel to the boundaries and the stability of a rotating orbit is not instantaneous in the parameter $\lambda$. In fact, there is an interval of angles containing $\pi / 4$ for which the map $\eta_{n} \rightarrow \eta_{n+2}$ is approximately linear, giving a continuum of periodic orbits. Figure 9 shows the intervals in $\eta$ for which the refraction map is approximately linear as a function of $\lambda$. Any map that contains an interval over which the map is linear has a continuum of periodic orbits for the angles associated with that interval. Figure 10a) and b) shows these periodic orbits for different values of $\lambda$. Figure 10c) shows the stable rotating periodic orbit associated with the equilibrium near $\eta=\pi / 4$.

The degenerate bifurcation at $\lambda=6$ in theory may well be a quick succession of multiple generic bifurcations manifesting itself as intermittency and very long transients. However, given the inherent noise level in the swarm, for all simulations we studied, close to $\lambda=6$ we had specular reflections and a continuum of periodic orbits. A better characterization of the bifurcation might be the following: For $\lambda<6$ the eigenvalues at the equilibria at $\eta=0$ and $\eta=\frac{\pi}{2}$ are always 1 , i.e. the equilibria are non-hyperbolic. At $\lambda=6$ the bifurcation changes the system from a conservative one (implying 


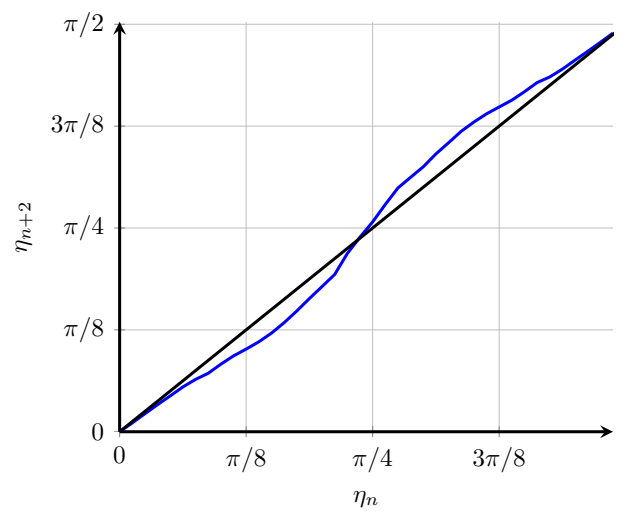

(a) $\lambda=3$

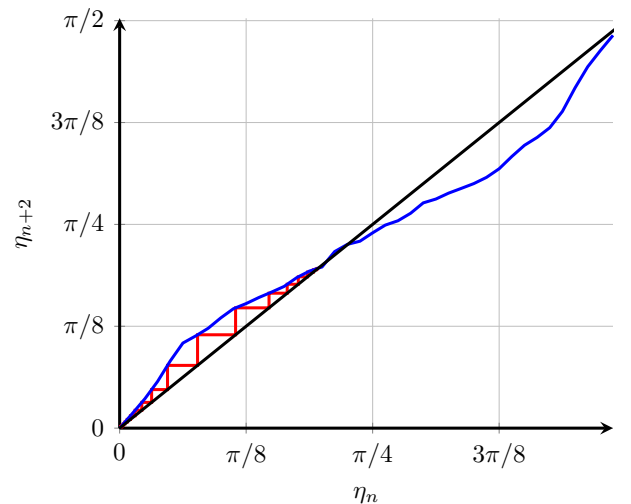

(c) $\lambda=10$

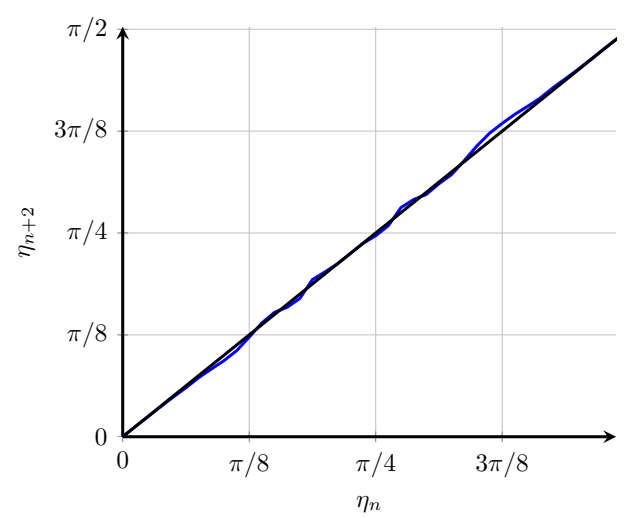

(b) $\lambda=6$

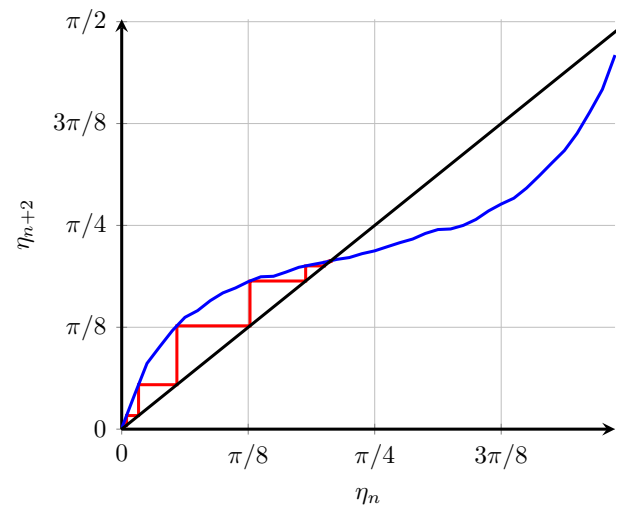

(d) $\lambda=25$

Figure 8: Mapping of the reflection angles $\eta_{n}$ to $\eta_{n+2}$ for swarms in a square for various $\lambda$ values. a) $\lambda=3$ : unstable fixed point at $\eta=\pi / 4$, stable fixed points at $\eta=0$ and $\pi / 2$. b) Linear map indicates specular reflection. c) and d) $\lambda \geq 10$ : the fixed points at $\eta=0, \pi / 2$ become unstable and a fixed point at $\eta<\pi / 4$ becomes stable. 


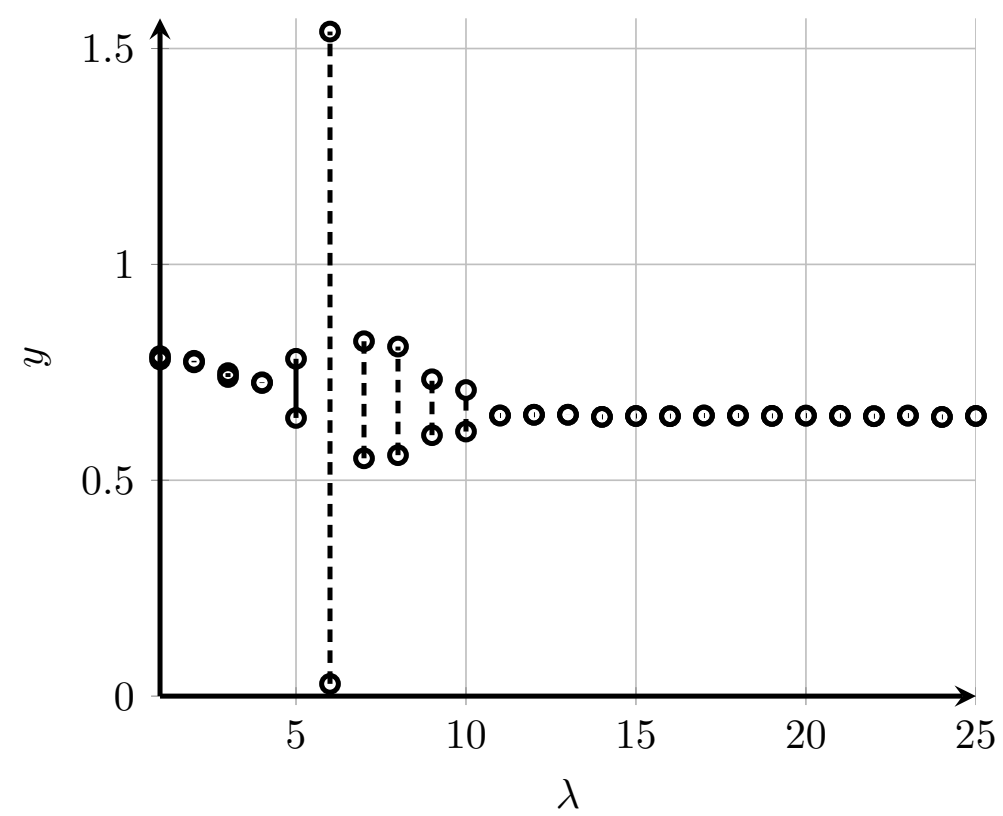

Figure 9: Fixed points for the iteration map of the square domain as a function of $\lambda$. For $4 \leq \lambda \leq 10$, the fixed points form a continuum.

non-hyperbolic fixed points) to a dissipative one generating hyperbolic fixed points.

When we analyze the dynamics of the iterated map, we rely on the simulation study of a swarm hitting a wall to generate the map discussed in section 2.1.2. Hence, the continuum of solutions indicated in figures 9 and 10 may not be all neutrally stable analytically. However, the interaction with the wall is a noisy process and depends on small variations in the initial conditions of the swarm particles. That noise level is overwhelming any small attraction or repulsion that may be analytically close to the bifurcation point. The situation is similar to the weakly stable transversal flow in the channel (figure 7).

\section{Full simulation of the swarm in a square.}

We numerically test our predictions based on the iterated refraction law. We perform two fundamental sets of experiments when simulating a flock set inside a $10 \times 10$ units square: i) we prepare an initial condition representing a flock solution of the attraction-repulsion model aligned with the walls of the square and ii) we prepare an initial condition representing a rotating flock solution. 


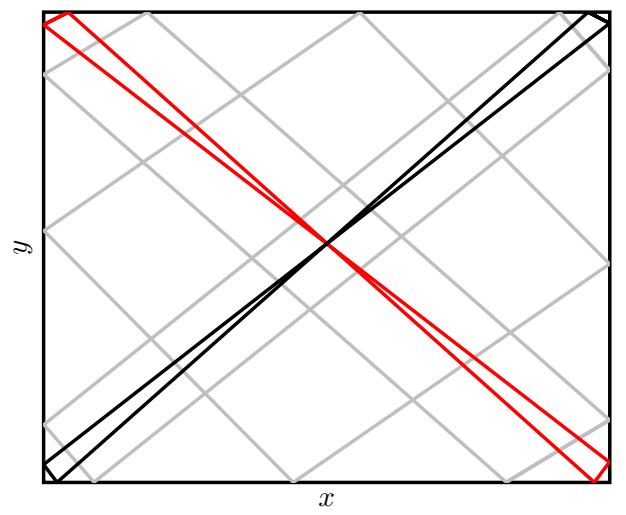

(a) $\lambda=7$

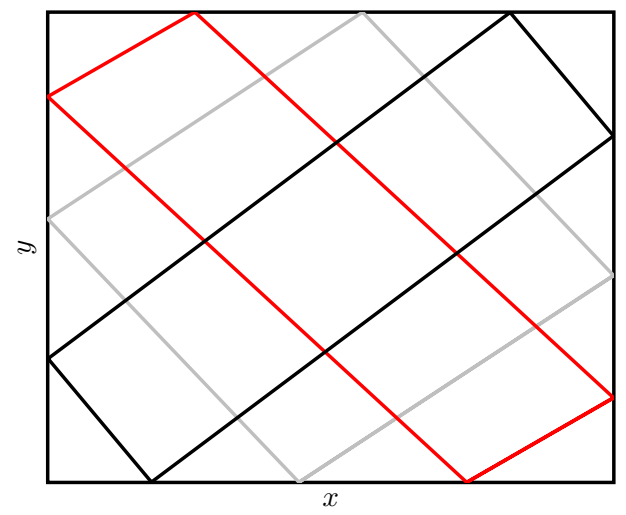

(b) $\lambda=9$

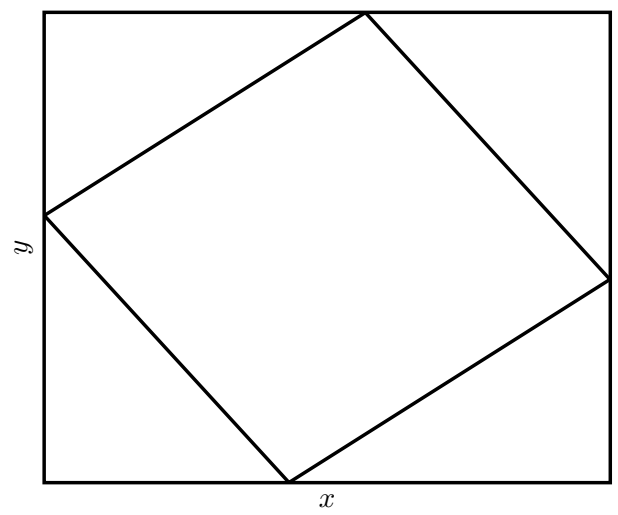

(c) $\lambda=11$

Figure 10: a,b): Trajectories, derived from the iterated maps, for the minimal (black) and maximal (red) fixed angles seen in figure 9. The grey trajectories indicate some of the periodic orbits in between the black and the red orbits. c): The single stable periodic orbit occurring for $\lambda=11$. 
We confirm the local stability results of the iterated map system when the attraction or repulsion to a fixed point is strong: For $\lambda<4$ the rotating solution is unstable while the solution parallel to the wall is stable. For $\lambda=25$ the rotating solution is stable and is depicted in figure 10c) while the flock moving parallel to a wall is unstable. However, the attraction to these fixed points is typically not global: Starting near the unstable rotating trajectory for small $\lambda$, the flock will disintegrate when it hits the corners of the square instead of transitioning to the stable orbit of a swarm parallel to the walls. Similarly, when the fixed points for the iterated maps are weakly stable, e.g. $4 \leq \lambda<7$, flocks mostly disintegrate.

Increasing $\lambda \geq 7$, we find a superposition of the neutrally stable solutions found via the iterative map. Figure 11a) shows the trajectory of the center of mass of a flock. It is strikingly similar to the set of neutrally stable cycles found via the iterated map analysis (figure 10a). Notice that there is a frequent change in the rotation direction with no preference on average for a clockwise or counterclockwise motion. As $\lambda$ increases, the set of neutrally stable periodic orbits shrinks. Comparing the center of mass trajectory in figure 10b) with figure 11b) again shows good agreement between the actual trajectory and the neutrally stable set of periodic orbits. Notice though that the two figures do not correspond to exactly the same values of $\lambda$ : While the transition region characterized in the iterated map by a continuum of periodic orbits can be found for $5 \leq \lambda \leq 10$ (see Figure 9), the transition region for the full swarm simulations (characterized by disintegrating flocks or by frequent changes of the direction of rotation) is found for $4 \leq \lambda \leq 14$.

Increasing $\lambda$ beyond 14, solutions cease to switch their rotation direction and move continuously from symmetry type $C_{2}$ corresponding to a trajectory that is symmetric under a rotation by $\pi$ (figure 11c)) to a symmetry type $C_{4}$ corresponding to a rotation by $\pi / 2$ (figure $11 \mathrm{~d})$ ).

\section{Internal Swarm Dynamics}

To gain further insight into the oscillating behavior seen in the kinetic energy (section 2.1), we perform a principal component analysis of the simulation data (see [20]). We consider the simulation as a series of $M$ snapshots $\mathbf{x}^{(k)}, k=1 . . M$ where $\mathbf{x}^{(k)}$ is a $4 N$ column vector containing the position and 


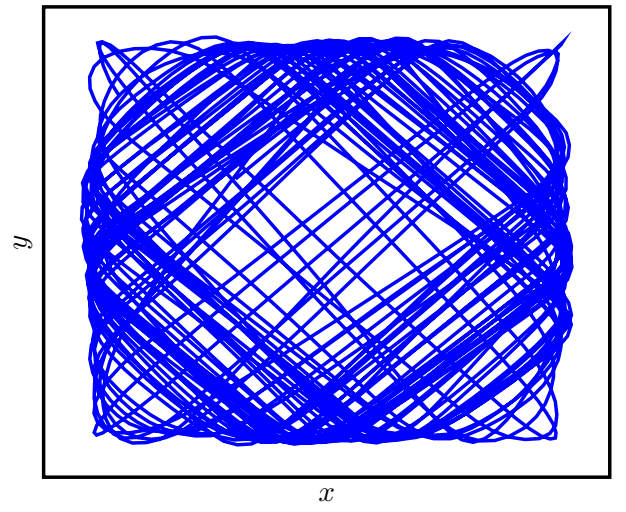

(a) $\lambda=7$

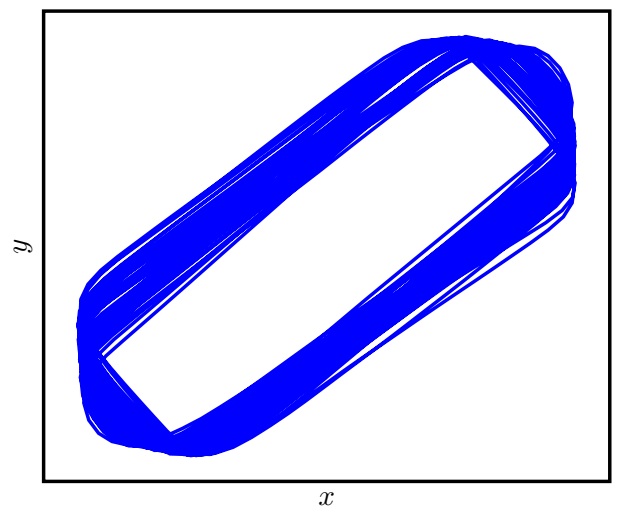

(c) $\lambda=18$

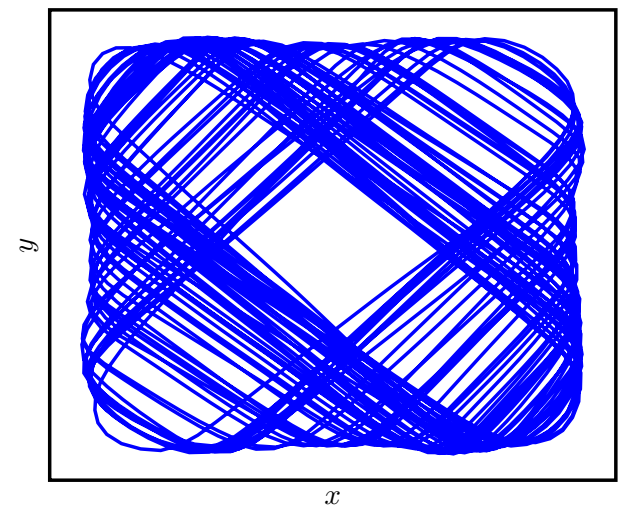

(b) $\lambda=15$

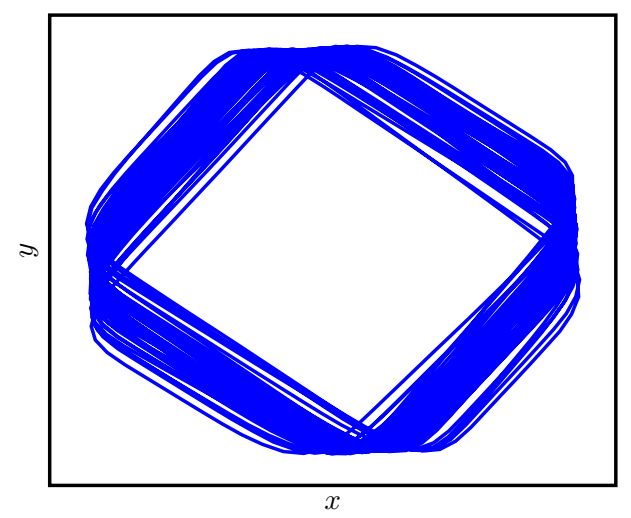

(d) $\lambda=25$

Figure 11: Center of mass trajectory for flocks set in a $10 \times 10$ box: typical trajectories as $\lambda$ increases. a) Trajectories rotate in both a clockwise and counterclockwise direction. b), c), and d) Trajectories only rotate in a clockwise direction. 
velocity of each particle at time $t_{k}$,

$$
\mathbf{x}=\left[\begin{array}{lllll}
x_{1}^{(1)} & \ldots & x_{1}^{(k)} & \ldots & x_{1}^{(M)} \\
& \ldots & & \ldots & \\
x_{4 N}^{(1)} & \ldots & x_{4 N}^{(k)} & \ldots & x_{4 N}^{(M)}
\end{array}\right]
$$

We subtract the center of mass from the position components and the mean velocity from the velocity components so that the ensemble is centered at 0. Principal component analysis seeks an orthonormal basis $\Phi$ that maximizes the mean squared projection of the data onto itself by solving the eigenvalue problem[20]

$$
C \phi=\gamma \phi
$$

where

$$
C=\left\langle\mathbf{x} \mathbf{x}^{T}\right\rangle=\frac{1}{M} \mathbf{x x}^{T}
$$

averages the space and velocity over time. Since the set of eigenvectors of $C$, $\Phi=\left\{\phi^{(j)}\right\}_{j=1 . . M}$ forms a basis for the field $\mathbf{x}^{(k)}$, we can write

$$
\mathbf{x}^{(k)}=\sum_{j=1}^{M} a_{j}^{(k)} \phi^{(j)}
$$

where the expansion coefficients $a_{j}$ are uncorrelated. We order the eigenvalues and the eigenvectors according to $\gamma_{1} \geq \gamma_{2} \geq \cdots \geq \gamma_{4 N} \geq 0$ implying that $\phi^{(1)}$ gives the optimal projection. Then the fraction of energy (variance) that each mode $i$ contributes to the total energy (variance) of the system is

$$
\frac{\sigma_{i}}{\sum_{i} \sigma_{i}} \quad \text { where } \quad \sigma_{i}=\sqrt{M} \sqrt{\gamma_{i}} .
$$

To generate the data for the principle component analysis, we initialize a flock with a horizontal heading for $\lambda=5$ and collect the spatial and velocity data from the moment the center of mass changes horizontal direction to time 100 seconds. We perform a principal component analysis described above and find that $67 \%$ of the energy is contained in the first mode shown in figure 12a. Note that the velocity components of $\Phi_{1}$ are very small and all the energy of the motion is in the positional pattern of the particles.

Projecting the data onto $\Phi_{1}$, we find an exponentially damped oscillation for the coefficient $a_{1}$ in equation (6), (Figure 12b). The oscillation in $a_{1}$ corresponds to a periodic scaling of the corresponding particle pattern creating 


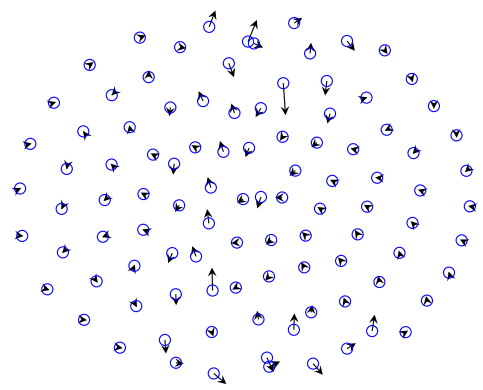

(a) first principal component

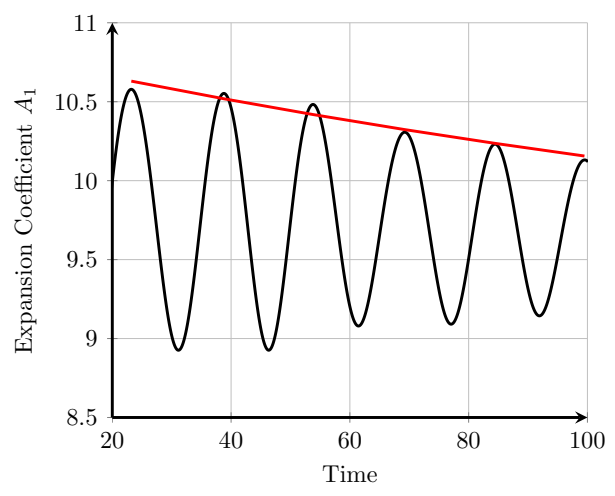

(b) time evolution of the amplitude

Figure 12: a) The first principle component of the oscillation of the swarm for $\lambda=5$. The particle positions are shown as circles, the velocities as blue arrows. Note that the velocities are very small. b) Time series of the amplitude of the first principle component. The red curve is an approximation for the decaying envelope of the oscillation.

a pulsating swarm. The fact that the velocity components in $\Phi_{1}$ are almost zero implies that the velocity of each particle is pretty much only in the radial direction. Any deviation from the pulsation shows up in the structure of the velocity components of the second principal component, which represents a small component of the energy of the motion.

\section{Swarm on swarm scattering}

We prepare two initial flocks satisfying Definition 1 as equilibrium solutions of the particle system (1) with initial velocity directions $\theta_{0} \in[0, \pi / 2)$ and positioned far enough apart that their interaction is negligible. We arrange the positions in such a way that the two flocks are moving with the velocities

$m_{L}=\sqrt{\alpha / \beta}\left[\cos \left(\theta_{0}\right), \sin \left(\theta_{0}\right)\right] \quad$ and $\quad m_{R}=\sqrt{\alpha / \beta}\left[-\cos \left(\theta_{0}\right), \sin \left(\theta_{0}\right)\right]$,

and hit each other at $x=0$.

We use a 4th order Adams-Bashforth-Moulton multi-step method to compute the numerical approximation to system (1). Otherwise, the simulation parameters are the defaults as discussed in section 1.2. 


\subsection{Two Particles}

To understand the basic interaction, we study the scattering of just two particles in a planar configuration i.e. each flock is represented by a single particle described by position vectors $\left(x_{i}, y_{i}\right)$ and velocity vectors $\left(v_{x_{i}}, v_{y_{i}}\right), i=1,2$.

The evolution equation Eq. (1) simplifies to the following eight equations

$$
\begin{array}{cc}
\dot{x}_{1}=v_{x_{1}} & \dot{y}_{1}=v_{y_{1}} \\
\dot{x}_{2}=v_{x_{2}} & \dot{y}_{2}=v_{y_{2}} \\
\dot{v}_{x_{1}}=\left(\alpha-\beta\left(v_{x_{1}}^{2}+v_{y_{1}}^{2}\right)\right) v_{x_{1}}-\frac{x_{1}-x_{2}}{r} \lambda U^{\prime}(r) \\
\dot{v}_{y_{1}}=\left(\alpha-\beta\left(v_{x_{1}}^{2}+v_{y_{1}}^{2}\right)\right) v_{y_{1}}-\frac{y_{1}-y_{2}}{r} \lambda U^{\prime}(r) \\
\dot{v}_{x_{2}}=\left(\alpha-\beta\left(v_{x_{2}}^{2}+v_{y_{2}}^{2}\right)\right) v_{x_{2}}+\frac{x_{1}-x_{2}}{r} \lambda U^{\prime}(r) \\
\dot{v}_{y_{2}}=\left(\alpha-\beta\left(v_{x_{2}}^{2}+v_{y_{2}}^{2}\right)\right) v_{y_{2}}+\frac{y_{1}-y_{2}}{r} \lambda U^{\prime}(r)
\end{array}
$$

with $r=\sqrt{\left(x_{1}-x_{2}\right)^{2}+\left(y_{1}-y_{2}\right)^{2}} \quad$ and $\quad U^{\prime}(r)=(-C / l \exp [-r / l]+\exp [-r])$. Defining the relative distance $X=x_{1}-x_{2}, Y=y_{1}-y_{2}$, the relative velocity $V_{x}=v_{x_{1}}-v_{x_{2}}, V_{y}=v_{y_{1}}-v_{y_{2}}$, and the average velocity $\bar{v}_{x}=1 / 2\left(v_{x_{1}}+v_{x_{2}}\right), \bar{v}_{y}=$ $1 / 2\left(v_{y_{1}}+v_{y_{2}}\right)$, the distance between the particles becomes $R=\sqrt{X^{2}+Y^{2}}$ and system (8) reduces to six equations

$$
\begin{aligned}
\dot{X} & =V_{x} \\
\dot{Y} & =V_{y} \\
\dot{V}_{x} & =\left(\alpha-\beta\left(\left(\bar{v}_{x}+V_{x} / 2\right)^{2}+\left(\bar{v}_{y}+V_{y} / 2\right)^{2}\right)\left(\bar{v}_{x}+V_{x} / 2\right)\right. \\
& -\left(\alpha-\beta\left(\left(\bar{v}_{x}-V_{x} / 2\right)^{2}+\left(\bar{v}_{y}-V_{y} / 2\right)^{2}\right)\left(\bar{v}_{x}-V_{x} / 2\right)-2 \frac{X}{R} \lambda U^{\prime}(R)\right. \\
\dot{V}_{y} & =\left(\alpha-\beta\left(\left(\bar{v}_{x}+V_{x} / 2\right)^{2}+\left(\bar{v}_{y}+V_{y} / 2\right)^{2}\right)\left(\bar{v}_{y}+V_{y} / 2\right)\right. \\
& -\left(\alpha-\beta\left(\left(\bar{v}_{x}-V_{x} / 2\right)^{2}+\left(\bar{v}_{y}-V_{y} / 2\right)^{2}\right)\left(\bar{v}_{y}-V_{y} / 2\right)-2 \frac{Y}{R} \lambda U^{\prime}(R)\right. \\
\dot{\bar{v}}_{x} & =\left(\alpha-\beta\left(\left(\bar{v}_{x}+V_{x} / 2\right)^{2}+\left(\bar{v}_{y}+V_{y} / 2\right)^{2}\right)\left(\bar{v}_{x}+V_{x} / 2\right)\right. \\
& +\left(\alpha-\beta\left(\left(\bar{v}_{x}-V_{x} / 2\right)^{2}+\left(\bar{v}_{y}-V_{y} / 2\right)^{2}\right)\left(\bar{v}_{x}-V_{x} / 2\right)\right. \\
\dot{\bar{v}}_{y} & \left.=\left(\alpha-\beta\left(\left(\bar{v}_{x}+V_{x} / 2\right)^{2}\right)\right)+\left(\bar{v}_{y}+V_{y} / 2\right)^{2}\right)\left(\bar{v}_{y}+V_{y} / 2\right) \\
& +\left(\alpha-\beta\left(\left(\bar{v}_{x}-V_{x} / 2\right)^{2}+\left(\bar{v}_{y}-V_{y} / 2\right)^{2}\right)\left(\bar{v}_{y}-V_{y} / 2\right)\right)
\end{aligned}
$$

\subsubsection{One-Dimensional Case}

Restricting our collision to a head on collision, we start the two particles with a distance of $\mu$ apart on the $x$-axis and with initial velocities $v_{x_{1}}=\sqrt{\alpha / \beta}, v_{x_{2}}=-v_{x_{1}}$. The average velocity remain at zero for all time and System (9) reduces to two equations where we assume, without loss of 
generality, $X>0$

$$
\begin{aligned}
\dot{X} & =V_{x} \\
\dot{V}_{x} & =\left(\alpha-\beta\left(V_{x}^{2} / 4\right)\right) V_{x}-2 \lambda U^{\prime}(X) .
\end{aligned}
$$

Equation (10) has an equilibrium at $(\hat{x}, 0)$ where $\hat{x}$ solves $U^{\prime}(X)=0$ with eigenvalues $\gamma_{1,2}(\alpha)=1 / 2\left(\alpha \pm \sqrt{\alpha^{2}-8 \lambda U^{\prime \prime}(\hat{x})}\right)$. Since $\alpha$ is positive, the equilibrium is always unstable.

It is possible to determine the global dynamics of the system by analyzing the nullclines of (10) and using the Poincaré-Bendixson theorem [24]. Since the equilibrium is unique and always unstable, there are only two possible cases for the $\omega$-limit sets: Trajectories either diverge to infinity or there exists a trapping region leading to a stable periodic orbit. Figure 13 shows direction fields and nullclines for the two cases. In the former all solutions go to infinity; whereas, in the latter there is a separatrix separating initial conditions with large relative velocities leading to solutions that diverge to infinity from initial conditions that have similar initial velocities that lead to periodic oscillations of the relative position and the relative velocity.

The analysis corresponds nicely to intuition. For small $\lambda$ the potential is never strong enough to trap the two particles, regardless of the initial velocity. If the potential is strong enough, particles, whose initial relative velocity is small, are captured and the relative distance between the two particles oscillate. For any $\lambda$ and any initial relative position, if the relative initial velocity is too large, the two particles move past each other and are not captured. The essential quantity is the time that the two particles spend within the reach of their attractive potential. For large velocities that time is too short to allow capture.

\subsubsection{Two Particles (Two-Dimensional)}

We consider the collision of two particles starting at a position $\left(x_{1}, y_{1}\right)=$ $(-\mu, 0)$ and $\left(x_{2}, y_{2}\right)=(\mu, 0)$ and moving upwards with the same speed and symmetric angles with respect to the vertical. Thus, they collide at $x=0$ and a vertical position determined by the scattering angle between them. The initial velocities are given by $v_{x_{1}}=-v_{x_{2}}$ and $v_{y_{1}}=v_{y_{2}}$, making the relative distance in $y$, the relative velocity in $y$, and the average velocity in $x, Y, V_{y}$, and $v_{x}$, respectively, zero for all time. System (9) then reduces to 


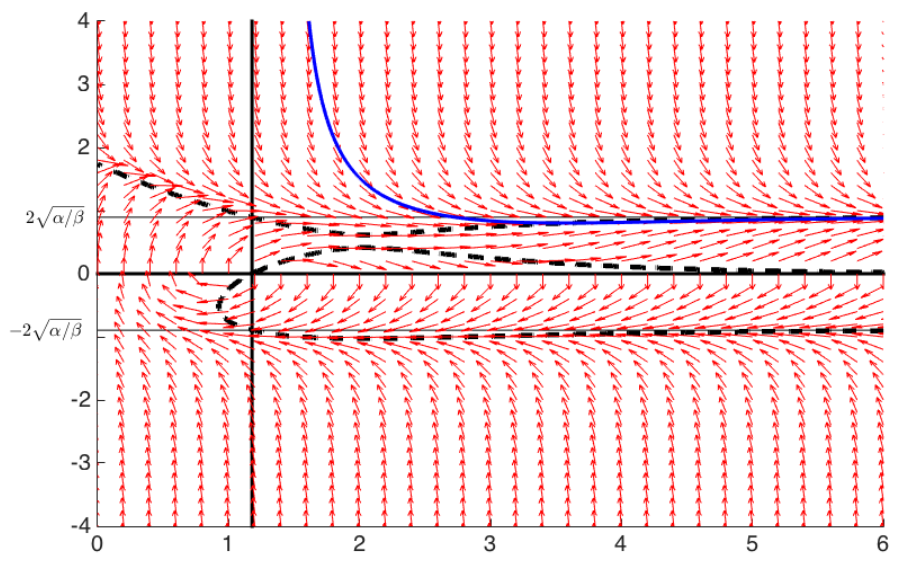

(a) $\lambda=5$

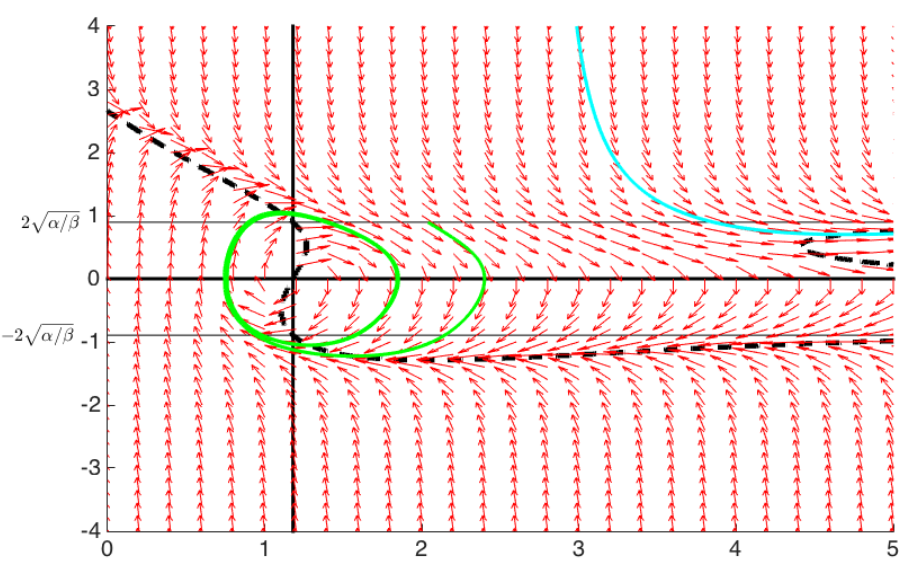

(b) $\lambda=22$

Figure 13: Phase diagram for the one-dimensional two particle model. The dashed lines represent isoclines, the full lines are trajectories. a) $\lambda=5$, all trajectories diverge, b) $\lambda=22$, there is a trapping region and a stable limit cycle. 


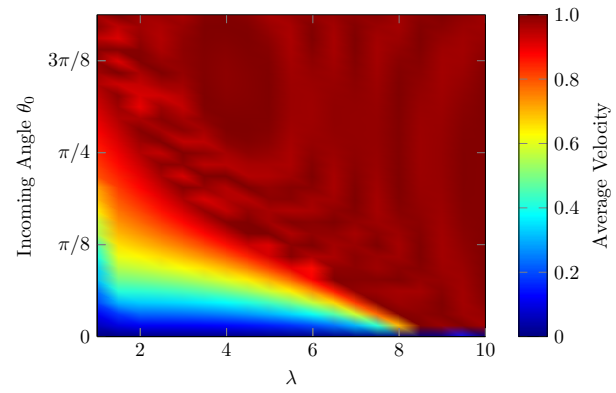

(a) Average Velocity $\bar{v}_{y}$

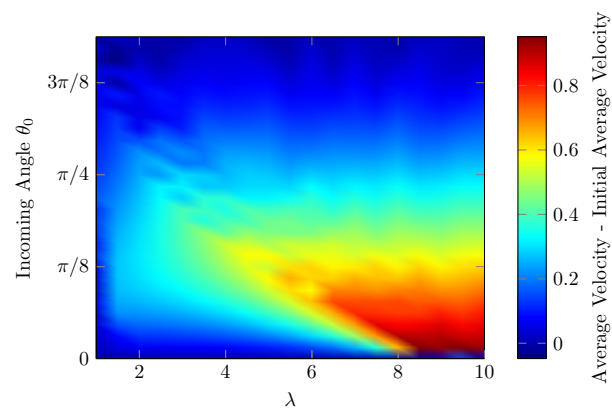

(b) Change in Average Velocity: $\bar{v}_{y}-\bar{v}_{y_{0}}$

Figure 14: a) Average velocity $\bar{v}_{y}$ after the collision of two interacting particles with various initial headings $\theta_{0}$ and potential pre-factors $\lambda$. An average velocity of zero implies that the two particles after collision head in different directions, ie. the paths diverge. An average velocity of one indicates that the two particles move together in the same direction. b) Change in average velocity: final average velocity $\bar{v}_{y}$ minus initial average velocity $\bar{v}_{y_{0}}$.

three differential equations

$$
\begin{aligned}
\dot{X} & =V_{x} \\
\dot{V}_{x} & =\left(\alpha-\beta\left(V_{x}^{2} / 4+\bar{v}_{y}^{2}\right)\right)\left(V_{x} / 2\right)+\left(\alpha-\beta\left(V_{x}^{2} / 4+\bar{v}_{y}^{2}\right)\right)\left(V_{x} / 2\right)-2 \frac{X_{1}}{\left|X_{1}\right|} \lambda U^{\prime}\left(\left|X_{1}\right|\right) \\
\dot{\bar{v}}_{y} & =1 / 2\left[\left(\alpha-\beta\left(V_{x}^{2} / 4+\bar{v}_{y}^{2}\right)\right) \bar{v}_{y}+\left(\alpha-\beta\left(V_{x}^{2} / 4+\bar{v}_{y}^{2}\right)\right) \bar{v}_{y}\right] .
\end{aligned}
$$

Setting $V=2 V_{x}$ and dropping the tilde for convenience, we rephrase system (11) in polar terms where $r^{2}=V^{2}+\bar{v}_{y}^{2}$ and $\zeta=\tan \left(\bar{v}_{y} / V\right)$ :

$$
\begin{aligned}
\dot{X} & =2 r \cos (\zeta) \\
\dot{r} & =r\left(\alpha-\beta r^{2}\right)-\lambda U^{\prime}(X) \cos (\zeta) \\
\dot{\zeta} & =1 / r \lambda U^{\prime}(X) \sin (\zeta)
\end{aligned}
$$

The steady state $(X, r, \zeta)=(\hat{x}, \sqrt{\alpha / \beta}, \pi / 2)$ describes two parallel particles moving with the equilibrium speed at a distance given by the minimum of the potential. It is linearly stable, with one negative real eigenvalue associated to $r$ and two purely imaginary eigenvalues associated to $X$ and $\zeta$. Performing a center manifold reduction we find that $r$ is of at least order two, leaving

$$
\begin{aligned}
\dot{X} & =0 \\
\dot{\zeta} & =\frac{\lambda}{2} \sqrt{\beta / \alpha} U^{\prime \prime \prime}(\hat{x}) X^{2}
\end{aligned}
$$

as the leading order terms on the CM. Since $U^{\prime \prime \prime}(\hat{x})<0$ the fixed point is 
asymptotically stable. However, the attraction is quadratic and very weak, indicating the system will take very long to settle.

To determine the scattering for arbitrary initial conditions, we study system (11) numerically for $(\alpha, \beta)=(1,5)$, various initial angles $\theta_{0}>0$ and various potential pre-factors $\lambda$. We choose initial conditions $\left(X_{0}, V_{x_{0}}, \bar{v}_{y_{0}}\right)=$ $\left(2,-2 \cos \theta_{0}, \sin \theta_{0}\right)$. Each simulation is ran for 500 time units. Figure 14 shows the average velocity in the $y$-direction $\bar{v}_{y}$ after the collision.

Our results match nicely to those analyzed in section 2.1. For small $\lambda$, the two particles do not influence each other enough to change the trajectory of both particles. As a result, they just pass each other and continue on their track Hence they do not reach the equilibrium of Eq. 12. As $\lambda$ increases, the potential gains enough strength to capture the two particles and the particles oscillate around a common heading. For almost head on collisions, i.e. $\theta_{0}$ small, the velocity in the x-direction is transferred to a velocity in the $y$-direction. As discussed, that oscillation decays algebraically until both particles move in parallel straight lines.

\subsection{Interacting Flocks}

Extending the observations made in the two particle system, we consider the full $N$ particle system Eq. (1). We initially set the center of mass of the left flock at the origin and the center of mass of the right flock at position $(5,0)$. The velocities are given by Eq. 7 with $\theta_{0} \in[0, \pi / 2)$. We characterize the degree of swarming using the polarization observable

$$
P(t)=\left|\frac{\sum_{i} v_{i}(t)}{\sum_{i}\left|v_{i}(t)\right|}\right| .
$$

Systems that settle into a flocking state will have high polarization $P \approx 1$, while two equal flocks on equal and opposite paths, as well as mills and double mills have $P \approx 0$.

As in the previous sections, we analyze the behavior of the system after the collision as a function of the potential pre-factor $\lambda$ and the collision angles $\theta_{0}$. Figure 15 shows the polarization after the collision and its change.

We find that the flocks act as quasi-particles and show different behavior for three regions in parameter space, which is very similar to the scattering of two particles.

1. For weak potential (i.e. small $\lambda$ ) and almost head on collisions (small $\left.\theta_{0}\right)$, the two flocks just pass each other and diverge. The short time 


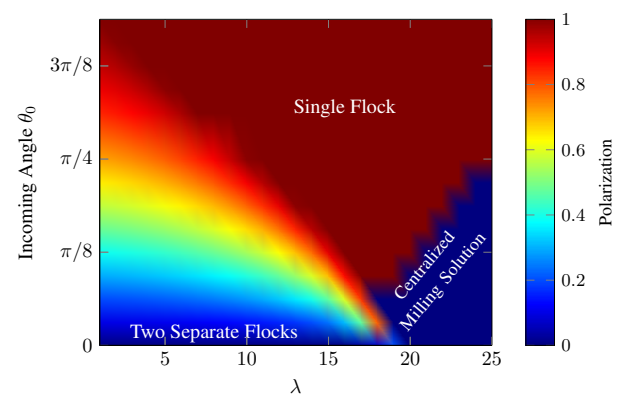

(a) Polarization

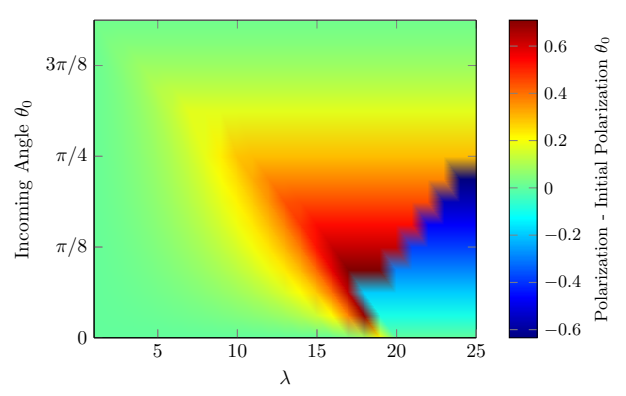

(b) Change in Polarization: Final - Initial

Figure 15: a) Polarization for two interacting flocks. b) Change in polarization: final polarization minus initial polarization. We run the simulation for 500 time units and average over the last 50 time units of the simulation.

interaction results in a small change of the departing angles towards each other but the change is not significant enough to merge the two flocks.

2. For glancing collisions ( $\theta_{0}$ large enough) and all $\lambda$, the two flocks merge and move as a single flock with uniform velocity.

3. For large $\lambda$ and almost head-on collisions, the two flocks generate a bound state in the form of a milling solution. The transition region between the single flock and the bound state shows unpredictable and complicated behavior - in addition to the milling solution, double mills and disintegrating flocks can be found.

\section{Discussion}

We study the attraction-repulsion model by simulating the impact of a flock on a flat, reflecting surface. We find that, although the model is not very sophisticated, the wall interactions have a high level of complexity that is tunable from hard specular reflection of the whole flock to inelastic oscillations that only graze the boundaries via a single parameter in the model. In addition, we find that the wall interaction creates new and previously not described internal excitations of the swarm.

Specifically, we find that if the dynamics of the flock is dominated by the kinetic terms (low potential strength), the reflection of the swarm is elastic 
and follows a specular reflection law for the flock represented as a quasiparticle. If the dynamics of the flock is determined by the potential term, the flock shows internal oscillations that lead to an inelastic refraction law at the boundary. A principal component analysis reveals that the internal oscillation is a radial pulsation akin to a breathing motion.

The inelastic scattering leads to a "stickiness" to the wall, producing a refraction law that tilts towards a motion of the swarm aligning to the wall. The resulting refraction map, which relates incoming and outgoing angles of the swarm, can be used as an iterated map to study multiple reflections of the swarm in a channel and in a square. Stable and unstable fixed points of the iterated refraction map are the associated stable and unstable directions of the flock. In a channel the attractor is the stable motion parallel to the channel, whereas, in a square there is a bifurcation from a stable motion parallel to the sides to a stable, rotating motion.

This bifurcation is degenerate: At bifurcation the refraction map becomes linear, representing exact specular reflection for a continuum of incoming angles that correspond to a continuum of oscillations that are neutrally stable. Comparing the analysis of the iterated maps with actual flock simulations, we found good agreement.

We extend the simulations for flock on wall scattering to flock on flock collisions. Reducing the flocks to two particle scattering, we analyze a sixdimensional dynamical system analytically. It can be shown that the two particles either diverge or form an oscillatory solution where they move in parallel but oscillate around a common direction. Simulations of scattering of full flocks show three qualitatively different dynamical regions, two of which are analogous to the two particles scattering: If the ratio of kinetic energy to potential energy is large, the paths of the two flocks cross, the angle between the two directions decreases but the two flocks still diverge. As the potential energy increases, the two flocks converge to a common direction but in an oscillatory way. For large enough potential energy and head on collisions, a bounded state appeared in the form of a milling solution.

Our analysis here extends the analysis of the Vicsek swarm in finite domains [2]. The interaction of the swarm with the boundary is similar for both models, i.e. a swarm typically aligns with the boundary and leads to similar periodic orbits in a square box. The details of the dynamical solutions are different and may be used to discriminate models and their experimental realization. In addition, since the Vicsek model is a first order model, no internal oscillations are possible which are a key feature of the inelastic 
behavior of the flock in the attraction-repulsion model.

It is intriguing to think about the analogy between swarms and particle physics, inherent in the attraction-repulsion model a bit further. Our study shows that the analogy extends to the concepts of internal excitations, scattering angles and bound states. This in particular leaves open the question of exciting internal swarm resonances leading to swarm fission.

\section{Acknowledgement}

All three authors gratefully acknowledge travel support through the KINet grant, NSF RNMS grant No. 1107291. D.A is supported by NSF grant DMS-1515592.

[1] I. Aoki., A simulation study on the schooling mechanism in fish., Bulletin of The Japanese Society of Fisheries Science, 48 (1982), pp. 10811088 .

[2] D. Armbruster, S. Motsch, And A. Thatcher, Swarming in bounded domains. Preprint 2016, Arizona State University.

[3] D. Armbruster, C. Ringhofer, And A. Thatcher, A kinetic model for an agent based market simulation, Networks and Heterogeneous Media, 10 (2015), pp. 527-542.

[4] J. Carrillo, M. D'orsogna, and V. Panferov, Double milling in self-propelled swarms from kinetic theory, Kinetic and Related Models, 2 (2009), pp. 363-378.

[5] J. Carrillo, Y. Huang, and S. Martin, Nonlinear stability of flock solutions in second-order swarming models, Nonlinear Analysis: Real World Applications, 17 (2014), pp. 332-343.

[6] J. Carrillo, S. Martin, and V. Panferov, A new interaction potential for swarming models, Physica D: Nonlinear Phenomena, 260 (2013), pp. 112-126.

[7] J. A. Carrillo, M. Fornasier, G. Toscani, and F. Vecil, Particle, kinetic, and hydrodynamic models of swarming, in Mathematical modeling of collective behavior in socio-economic and life sciences, Springer, 2010, pp. 297-336. 
[8] J. A. Carrillo, Y. Huang, And S. Martin, Explicit flock solutions for quasi-morse potentials, European Journal of Applied Mathematics, 25 (2014), pp. 553-578.

[9] A. Cavagna, I. Giardina, A. Orlandi, G. Parisi, A. ProcacCini, M. Viale, AND V. ZdRaVkovic, The starflag handbook on collective animal behaviour: 1. empirical methods, Animal Behaviour, 76 (2008), pp. 217-236.

[10] Y.-L. Chuang, M. R. D’orsogna, D. Marthaler, A. L. Bertozzi, And L. S. Chayes, State transitions and the continuum limit for a 2d interacting, self-propelled particle system, Physica D: Nonlinear Phenomena, 232 (2007), pp. 33-47.

[11] I. D. CouzIn And J. KRAuse, Self-organization and collective behavior in vertebrates, Advances in the Study of Behavior, 32 (2003), pp. 1-75.

[12] F. Cucker And S. Smale, Emergent behavior in flocks, Automatic Control, IEEE Transactions on, 52 (2007), pp. 852-862.

[13] A. Czirók, A.-L. Barabási, And T. Vicsek, Collective motion of self-propelled particles: Kinetic phase transition in one dimension, Physical Review Letters, 82 (1999), p. 209.

[14] M. R. D’Orsogna, Y.-L. Chuang, A. L. Bertozzi, and L. S. CHAYES, Self-propelled particles with soft-core interactions: patterns, stability, and collapse, Physical review letters, 96 (2006), p. 104302.

[15] R. C. Fetecau, Y. Huang, and T. Kolokolnikov, Swarm dynamics and equilibria for a nonlocal aggregation model, Nonlinearity, 24 (2011), p. 2681.

[16] S.-Y. HA AND E. TADMOR, From particle to kinetic and hydrodynamic descriptions of flocking, arXiv preprint arXiv:0806.2182, (2008).

[17] R. Hegselmann and U. Krause, Opinion dynamics and bounded confidence: models, analysis and simulation, J. Artificial Societies and Social Simulation, 5 (2002).

[18] D. Helbing, L. Buzna, A. Johansson, And T. Werner, Selforganized pedestrian crowd dynamics: Experiments, simulations, and design solutions, Transportation science, 39 (2005), pp. 1-24. 
[19] A. Huth And C. Wissel, The simulation of the movement of fish schools, Journal of theoretical biology, 156 (1992), pp. 365-385.

[20] M. KIRBY, Geometric data analysis: an empirical approach to dimensionality reduction and the study of patterns, John Wiley \& Sons, Inc., 2000 .

[21] H. Levine, W.-J. Rappel, And I. Cohen, Self-organization in systems of self-propelled particles, Physical Review E, 63 (2000), p. 017101.

[22] A. Mogilner, L. Edelstein-Keshet, L. Bent, And A. Spiros, Mutual interactions, potentials, and individual distance in a social aggregation, Journal of mathematical biology, 47 (2003), pp. 353-389.

[23] C. W. Reynolds, Flocks, herds and schools: A distributed behavioral model, in ACM Siggraph Computer Graphics, vol. 21, ACM, 1987, pp. 25-34.

[24] A. Thatcher, Swarming in Bounded Domains, PhD thesis, Arizona State University, 2015.

[25] T. Vicsek, A. Czirók, E. Ben-Jacob, I. Cohen, And O. SHochet, Novel type of phase transition in a system of self-driven particles, Physical review letters, 75 (1995), p. 1226. 